\title{
Über die aus der Einwirkung von Blausäure auf ungesättigte Aldehyde hervorgehenden Verbindungen
}

\author{
(II. Mittheilung) \\ von
}

Gustav Johanny.

Aus dem ehemischen Laboratorium des Prof. Ad. Lieben an der k. k. Universität in Wien.

(Vorgeiegt in der Sirzung am 2l. Juni 1894.)

Vor längerer Zeit habe ich gezeigt, ${ }^{1}$ auf welche Art die Einwirkung von Cyanwasserstoffsäure auf Methyläthylacroleïn zu dem Amid einer ungesättigten Oxysäure der Heptylreihe geführt hat. Es war mir damals nur möglich das Calciumsalz dieser Säure, der $\alpha$-Oxy-ß-Propyliden-n-Buttersäure, in eben für die Analyse ausreichender Menge darzustellen, auf die Isolirung der Säure selbst musste ich verzichten.

Nachdem ich in der Folge eine grössere Menge Säureamid auf dem beschriebenen umständlichen Wege dargestellt hatte, wurde dasselbe mit Ätzbaryt zersetzt, aus dem krystallisirten Bariumsalz die gesuchte ungesättigte Oxysäure $\mathrm{C}_{\hat{\imath}} \mathrm{H}_{12} \mathrm{O}_{3}$ erhalten und diese durch die Elementaranalyse und die Bromaddition identificirt. Auch in dem mit zwei Moleküllen Wasser krystallisirenden Zinksalz bestätigte die Zinkbestimmung die für die Säure durch die Elementaranalyse gefundenen Werthe.

Da die in Rede stehende Säure in der $\beta \gamma$-Stellung doppelt gebundene Kohlenstofiatome hat, versuchte ich die von Rud. Fittig angegebene Umwandlung der Dibromide solcher Säuren

I Monatshefte für Chemie, XI, 399 ff. 
zu Lactonen und Ketonsäuren durchzuführen. Ich erhielt aus Chloroformlösung das Dibromid krystallisirt und aus demselben mittelst Natriumcarbonat das entsprechende ebenfalls krystallisirte Bromlacton. Da hiebei unter Abspaltung von Ameisensäure wieder das Ausgangsmaterial, nämlich Methyläthylacroleïn entstand, war die Reaction bis zur Bildung von Oxylacton und $\gamma$-Ketonsäure nicht durchzuführen.

Auch die gesättigte Säure konnte ich nicht darstellen. Natriumamalgam, Zink in saurer und in ammoniakalischer Lösung wirkten auf die ungesättigte Säure gar nicht ein, Jodwasserstoffsäure reducirte unter Abspaltung von Ameisensäure bis zum gesättigten Kohlenwasserstoff $\mathrm{C}_{6} \mathrm{H}_{14}$, beziehungsweise dem Jodid desselben.

Das Bariumsalz, das Dibromid und das Bromlacton der ungesättigten Säure lieferten gut ausgebildete, messbare Krystalle. Die Krystalle des Säureamid liessen nur eine annähernde Messung zu.

Herr Ad. Stengel hatte die Freundlichkeit, die krystallographische Bestimmung dieser Körper im mineralogischen Institut des Herrn Prof. A. Schrauf auszuführen, wofür ich ihm auch an dieser Stelle besten Dank sage.

Das Amid der $\alpha$-Oxy- $\beta$-Propyliden- $n$-Buttersäure wurde auf die in der ersten Mittheilung beschriebene Art durch Verseifung des acetylirten Cyanides mit rauchender Salzsäure dargestellt und wiederholt aus Alkoholäther umkrystallisirt.

Herr Ad. Stengel theilt mir über dasselbe Folgendes mit:

Krystallsystem: triklin?

Die untersuchte Substanz krystallisirt in dünnen Plättchen mit rhombischem Querschnitte von $124^{\circ}$ und stark corrodirten Seitenflächen $\left(\infty P_{1} P\right)$, die eine verlässliche Bestimmung des Krystallsystems nicht zulassen. Die Substanz ist zweiaxig.

Um das Bariumsalz der ungesättigten Säure darzustelien, wurde das Amid mit Ätzbaryt mehrere Stunden lang am Rückflusskühler erhitzt und dann so lange von der Lösung abdestillirt, als noch Ammoniak fortging. Nach Abfiltriren vom überschüssigen Bariumhydroxyd wurde in die zum Kochen 
erhitzte Lösung Kohlensäure eingeleitet, Baryumcarbonat abgetrennt und mit heissem Wasser nachgewaschen. Die wässerige Lösung des Baryumsalzes schied nach Einengen am Wasserbade säulenförmige, winkelig aneinander gelagerte Krystallstöcke $a b$.

Die aus Wasser umkrystallisirte Substanz wurde krystallographisch bestimmt:

Krystallsystem: rhombisch.

Axenverhältniss $a: b: c=1 \cdot 0296: 0 \cdot 9369: 1$.

An den leistenförmig ausgebildeten Plättchen wurden folgende Flächen beobachtet: $O P$ vorherrschend, $1 /{ }_{4} \bar{P} \infty, \infty P, \breve{P} 2$ $2 \breve{P} 2,4 \breve{P} 2$.

Die wichtigsten Winkelwerthe sind:

$$
\begin{aligned}
& \infty P: \infty P^{\prime}=84^{\circ} 35^{\prime} \\
& O P: 2 \widetilde{P} 2=11417 .
\end{aligned}
$$

Die Plättchen zeigen gerade Auslöschung.

Das Baryumsalz ist in Alkohol sehr schwer, in Wasser sehr leicht löslich und krystallisirt aus der wässerigen Lösung mit 3 Molekülen Wasser.

Zur Analyse wurde das Salz wiederholt aus Wasser umkrystallisirt, zerrieben und zwischen Filtrirpapier trocken gepresst.

0.6862 g Substanz verloren, bei $97^{\circ}$ im Meyer'schen Luftbad zum constanten Gewicht erhitzt, $0.0760 \mathrm{~g}$, d. i. $11.08 \%$. Für $\mathrm{Ba}\left(\mathrm{C}_{i} \mathrm{H}_{11} \mathrm{O}_{3}\right)_{2}+3 \mathrm{H}_{2} \mathrm{O}$ wären 3 Moleküle Krystallwasser mit $11.32 \%$ berechnet.

$0.9727 \mathrm{~g}$ bei $97^{\circ}$ getrocknetes Salz wurden in Wasser gelöst und verbrauchten zur Ausfällung des Baryums $22 \cdot 4 \mathrm{~cm}^{3}$ $\mathrm{H}_{2} \mathrm{SO}_{4}$ vom Titer 0.010086 , also $0.2258264 \mathrm{~g} \mathrm{H}_{2} \mathrm{SO}_{4}$, welche $0 \cdot 315696 \mathrm{~g} \mathrm{Ba}$ entsprechen (I).

Das ausgeschiedene $\mathrm{BaSO}_{4}$ betrug $0.5381 \mathrm{~g}$, entsprechend $0 \cdot 313698 \mathrm{~g}$ Ba (II).

In 100 Theilen:

$$
\overbrace{\text { I. }}^{\text {Ga } \ldots . .32 \cdot 47} \text { II. }_{32 \cdot 25} \underbrace{\begin{array}{l}
\text { Berechnet für } \\
\mathrm{Ba}\left(\mathrm{C}_{7} \mathrm{H}_{11} \mathrm{O}_{3}\right)_{2}
\end{array}}_{32 \cdot 39}
$$


Die wässerige Lösung des reinen Baryumsalzes wurde mit der zur Abscheidung der Säure nöthigen Menge Schwefe!säure versetzt, vom Bariumsulfat abfiltrirt und die wässerige Lösung der

\section{a-Oxy--3-Propyliden-11-Buttersäure}

mit Äther ausgeschüttelt. Nach Abdestilliren des Äthers hinterblieb eine dickliche, schwach gelblich gefärbte Flüssigkeit, welche, an der Luft stehend, keine Krystalle abschied, woh1 aber im Exsiccator über Chlorcalcium krystallinisch erstarrte und sich durch die Analyse als die gesuchte Süure erwies.

$0.2267 \mathrm{~g}$ Substanz, welche im Exsiccator über $\mathrm{CaCl}_{2}$ constant geworden und zu schneeweissen, dicht ancinande! gelagerten Blättchen crstart war, ergab, mit Bleichromat verbrannt, $0 \cdot 48+1 g \mathrm{CO}_{2}$ und $0 \cdot 1711 \mathrm{~g} \mathrm{H}_{2} \mathrm{O}$.

In 100 Theilen:

$$
\begin{aligned}
& \text { Berechnet } \\
& \text { Gefunden für } \mathrm{C}_{7} \mathrm{Il}_{1} \mathrm{O}_{\mathrm{:}} \\
& \text { C..... } 58 \cdot 24 \quad 58 \cdot 33 \\
& \text { H ..... } 8.39 \quad 8 \cdot 33
\end{aligned}
$$

Zur Charakterisirung als ungesüttigten Körper wurde die Addition von Brom vorgenommen:

I. $0.6335 \mathrm{~g}$ Säure, im Exsiccator constant und krystallinisch geworden, wurden in Eisessig. gelöst und verbrauchten $0.7105 \mathrm{~g}$ bis zur bleibenden Färbung durch überschüssiges Brom. Theoretisch hätte $0.7038 \mathrm{~g}$ Brom verbraucht werden sollen.

II. $1.31 \mathrm{~g}$ krystallisirter Säure in Eisessiglösung brauchten bis zur Bromfärbung $1.453 \mathrm{~g}$ Brom. Die Theorie fordert $1.454 \mathrm{~g}$ Brom zur Sättigung.

Die Säure krystallisirt in schneeweissen Blättchen, welche an der Luft schnell Feuchtigkeit anziehen und zu einer öligen Masse zerfliessen. Im Exsiccator über Chlorcalcium bilden sich die Krystalle wieder. Der Schmelzpunlit wurde in geschiossener Capillare bei $43^{\circ}$ gefunden und blieb unverändert beim noch- 
maligen Schmelzen der wieder erstarrten Substanz. Denselben Schmelzpunkt zeigen auch die aus Alkohol erhaltenen Säurekrystalle. In Wasser, Alkohol, Äther, Chloroform, Benzol, Eisessig ist die Säure leicht löslich, mit Wasserdämpfen nur wenig flüchtig.

Aus der reinen Säure wurde das Zinksalz durch Digeriren und späteres Erhitzen ihrer wässerigen Lösung mit Zinkcarbonat dargestellt. Es krystallisirt in kleinen, zu Büscheln vereinigten Nädelchen, welche in heissem Wasser leicht löslich sind und 2 Moleküle Krystallwasser enthalten.

$0 \cdot 4145 \mathrm{~g}$ Zinksalz, aus Wasser umkrystallisirt, zerrieben und zwischen Filtrirpapier trocken gepresst, erlitten bei $97^{\circ}$ zum constanten Gewicht erhitzt, einen Verlust von $0.0969 g$, d. i. $8.90 \%$. Der Gehalt an Krystallwasser in $\mathrm{Zn}\left(\mathrm{C}_{7} \mathrm{H}_{11} \mathrm{O}_{3}\right)_{2}-{ }_{-}$ $+2 \mathrm{H}_{2} \mathrm{O}$ berechnet sich mit $9 \cdot 30 \%$.

$0.6821 \mathrm{~g}$ Substanz, bei $97^{\circ}$ bis zum constanten Gewicht getrocknet, ergaben $0.1562 \mathrm{~g} \mathrm{ZnO}$ (gefältt als basisches Carbonat), entsprechend $0 \cdot 12534 \mathrm{~g} Z \mathrm{Zn}$.

In 100 Theilen:

$$
\mathrm{Zn}_{1 .} \ldots \underbrace{\text { Gefunden }}_{18 \cdot 38} \quad \frac{\begin{array}{c}
\text { Berechnet für } \\
\mathrm{Zn}\left(\mathrm{C}_{1} \mathrm{H}_{11} \mathrm{O}_{3}\right)_{2}
\end{array}}{18 \cdot 52}
$$

Aus der Eisessiglösung der bromirten Säure wurde versucht das

\section{Dibromid der Säure}

darzustellen durch Stehenlassen im Vacuum über Ätzkali. Nach $2 \pm$ Stunden war fast alles Lösungsmittel verdunstet und eine ölige, etwas gelblich gefärbte Masse zurücligeblieben. Mit wenigen Tropfen Wasser versetzt, schieden sich im Vacuum über Kali alsbald feine, zu Büscheln vereinigte Nadeln in der öligen Masse aus, welche sofort abgesaugt wurden. Gleichzeitig mit der Krystallausscheidung war deutliche Entwicklung von Bromwasserstoff sichtbar und im Filtrat Bromwasserstoff nachzuweisen. Die abgesaugten Krystalle schmolzen nach Umkrystallisiren aus Alkohol zwischen 95 und $98^{\circ}$ und enthielten $41.47 \%$ Brom, während das Dibromid der Säure $52.63 \%$ und 
$\mathrm{C}_{7} \mathrm{H}_{11} \mathrm{O}_{3} \mathrm{Br}$ nur $35 \cdot 87 \%$ Brom theoretisch verlangt. Schon der wenig scharfe Schmelzpunkt wies auf eine unreine Substanz, beziehungsweise auf ein Gemenge hin.

Gelegentlich eines zweiten Versuches, aus der bei der Bromirung der ungesättigten Säure erhaltenen Eisessiglösung das Dibromid zu isoliren, wurde Wasser sorgfältig vermieden. Jedoch konnten die letzten Reste des Lösungsmittels nicht gut entfernt werden, und als durch einen unglücklichen Zufall die ölige Masse feucht wurde, Bromwasserstoffentwicklung auftrat und ein dem aus dem früheren Versuch erhaltenen ähnliches Product ergab, wurde weiterhin trockenes Chloroform als Lösungsmittel verwendet, weil dieses durch Durchsaugen von trockener Luft leicht entfernt werden konnte.

$5.103 g$ ungesättigte Säure wurden in beiläufig $30 \mathrm{~cm}^{3}$ trockenem Chloroform gelöst und unter Kühlung mit Brom versetzt. Obwohl anfangs nur wenig Brom zugesetzt worden war, blieb die Flüssigkeit gleich braun gefärbt. Weiteres Brom wurde nach und nach zugesetzt, bis die theoretisch nothwendige Menge von $5 \cdot 66 \mathrm{~g}$ überschritten war. Nachdem der Überschuss an Brom und das Lösungsmittel durch Durchsaugen von mit Schwefelsäure und Chlorcalcium getrockneter Luft zum grössten Theil entfernt worden war, erstarrte der Rückstand krystallinisch. Nach wiederholtem Umkrystallisiren aus Chloroform (und theils auch aus Benzol) wurden säulenförmige Krystalle erhalten, deren Schmelzpunkt bei 124-125 liegt. Die gehscmolzene Substanz erstarrte nicht mehr und war violett gefärbt.

Über das krystallographische Verhalten theilte mir Herr Ad. Stengel mit:

Krystallsystem: triclin.

Axenverhältniss: $a: b: c=1 \cdot 2923: 1 \cdot 7737: 1$.

$$
\begin{aligned}
& \Varangle b a c=77^{\circ} 56^{\prime} \\
& \Varangle a b c=8646 \\
& \Varangle a c b=8855
\end{aligned}
$$

Säulenförmig ausgebildete Krystalle mit den acht Flächen der Combination $\infty P$ und $\bar{P} \infty$. 
Die wichtigsten Winkelwerthe sind:

$$
\begin{aligned}
\infty P: \infty^{\prime} P & =108^{\circ} 11^{\prime} \\
\infty P: \bar{P}^{\prime} \infty & =12713 \\
\infty^{\prime} P: \bar{P}^{\prime} \infty & =11435
\end{aligned}
$$

Spaltbar nach $\infty P^{\prime}$.

Die wiederholt aus Chloroform umkrystallisirte Substanz wurde zur Elementaranalyse und zur Brombestimmung verwendet:

I. $0 \cdot 2190 \mathrm{~g}$ zerriebener und im Exsiccator über Chlorcalcium constant gebliebener Krystalle ergaben, mit Bleichromat und vorgelegter Silberspirale verbrannt, $0.2229 \mathrm{~g} \mathrm{CO}_{2}$ und $0.0792 \mathrm{~g} \mathrm{H}_{2} \mathrm{O}$.

II. $0 \cdot 2986 g$ Substanz gaben, mit Ätzkalk verbrannt, $0.3657 \mathrm{~g}$

\begin{tabular}{|c|c|c|}
\hline \multicolumn{2}{|c|}{ Gefunden } & \multirow{2}{*}{$\begin{array}{r}\text { Berechnet } \\
\text { für } \mathrm{C}_{7} \mathrm{H}_{32} \mathrm{O}_{3} \mathrm{Br}_{2}\end{array}$} \\
\hline I. & II. & \\
\hline$\ldots \ldots 27 \cdot 75$ & $\ldots$ & $27 \cdot 63$ \\
\hline$\ldots .4 \cdot 02$ & - & $3 \cdot 95$ \\
\hline$\ldots \ldots \ldots-$ & $52 \cdot 39$ & $52 \cdot 63$ \\
\hline
\end{tabular}
$\mathrm{AgBr}$.

In 100 Theilen:

Die erhaltenen Resultate berechtigen, den Körper als das reine Dibromid der ungesättigten Säure $\mathrm{C}_{3} \mathrm{H}_{12} \mathrm{O}_{3}$ anzusprechen

\section{Bromlacton.}

Vor einiger Zeit hat Rud. Fittig ${ }^{1}$ gezeigt, dass aus den Dibromiden von $\beta \gamma$-ungesättigten Säuren beim Kochen mit Wasser oder beim Stehenlassen in schwach alkalischer Lösung unter Austritt von Bromwasserstoff zunächst Bromlactone und aus diesen durch weitere Abspaltung von Bromwasserstoff Oxylactone und daneben $\gamma$-Ketonsäuren entstehen.

$D a$ die vorliegende $\alpha$-Oxy- $\beta$-Propyliden- $n$-Buttersäure ihrer Entstehung nach ebenfalls eine $\beta \gamma$-ungesättigte Säure ist, so

1 Annalen der Chemie, 268, 50. 
schien es mir angezeigt, diese Fittig'sche Reaction auszuführen, umsomehr, als ich, wie schon früher erwähnt, gelegentlich der Versuche das Dibromid aus der Fisessiglösung der bromirten Säure darzustellen, unter Entwicklung von Bromwasserstoff einen krystallisirten Körper erhalten hatte, dessen Gehalt an Brom sich einem Körper $\mathrm{C}_{7} \mathrm{H}_{11} \mathrm{O}_{3} \mathrm{Br}$ näherte.

Es selang mir zwar, das gebromte Lacton darzustellen, jedoch die Reaction im Sinne Fittig's weiter durchzuführen war nicht möglich, weil Zersetzung eintrat unter Bildung von Nethyläthylacroleïn.

$2 \cdot 10$ s Dibromid wurde mit $50 \mathrm{~cm}^{3}$ Wasser übergossen und eine Lüsung von $0.55 \mathrm{~g}$ Soda in $10 \mathrm{~cm}^{3}$ Wasser zugefügt. Nach liurzer Zeit war das Dibromid vollständig in Lösung gegangen, gleichzeitig aber begann sich die Flüssigkeit zu trüben, und Ötröpfchen schieden sich am Boden des Kölbchens ah. Fine spontane Erwärmung der Flüssigkeit war nicht wahrzunehmen, aber der intensive Geruch nach Methyläthylacroleïn war gleich beim Beginn der auftretenden Trübung ganz deutlich. Dic alkalische Flüssigkeit wurde wiederholt mit Äther ausgeschüttelt und die atherische Lösung mit wenig Natriumcarbonat grewaschen. Nachden der Äther zum grössten Theil aus dem Wasserbade abdestillirt worden war, begannen sich in der erkaltenden Fjüssigkeit flache, tafelförmige Krystalle auszuscheiden. Die gelblich gefärbte, ölige Mutterlauge ergab keine weiteren Krystalle, verursachte auf Papier einen durchscheinenden Fleck und war dem charakteristischen Geruche nach Methyläthylacrolë̈n. Die Menge dessciben war zu gering, als dass es auch durch den Siedepunkt hätte identificirt werden können. Die Krystalle wurden durch Abpressen zwischen Filtrirpapier von dem anhängenden Methyläthylacroleïn befreit und wiederholt theils aus Chloroform, theils aus Ather umkrystallisirt. Die aus Chloroform, wie auch die aus Äther erhaltenen Krystalle haben die gleiche Gestalt, schmelzen bei $82-83^{\circ}$ und zeigen, nach dem Erstarren wieder geschmolzen, denselben Schmelzpunkt.

Die Ausbeute blieb weit hinter der theoretischen zurück. Aus $2 \cdot 1 \mathrm{~g}$ Dibromid wurden $0.42 \mathrm{~g}$ Bromlacton, also $27 \%$ der theoretischen Ausbeute erhalten. 
Die krystallographische Bestimmung ergab:

Krystallsystem: monoklin.

Axenverhältniss: $a: b: c=0 \cdot 7938: 0 \cdot 6788: 1$.

$$
\Varangle a b c=75^{\circ} 10^{\prime} .
$$

An den tafelartig, parallelschalig gebauten Krystallen treten auf vorherrschend $O P$ und $\infty P,+P,-P, P_{\infty},{ }^{2} / 3 P_{\infty}$.

Die wichtigsten Winkelwerthe sind:

$$
\begin{aligned}
& O P:+P=125^{\circ} 13^{\prime} \\
& +P: \infty P=15433 \\
& +P:{ }^{\prime} P=103 \quad 14
\end{aligned}
$$

Die zur Analyse verwendeten Krystalle wurden zerrieben und einige Tage im Exsiccator über Chlorcalcium belassen. Das Gewicht hatte sich nicht geändert.

I. $0 \cdot 1754 g$ Substanz ergab mit Bleichromat und vorgelegter Silberspirale verbrannt, $0.2415 \mathrm{~g} \mathrm{CO}_{2}$ und $0.0810 \mathrm{~g} \mathrm{H}_{2} \mathrm{O}$.

\begin{tabular}{|c|c|c|c|}
\hline & \multicolumn{2}{|c|}{ Gefunden } & \multirow{2}{*}{$\begin{array}{r}\begin{array}{r}\text { Berechnet } \\
\text { für } \mathrm{C}_{7} \mathrm{H}_{11} \mathrm{O}_{3} \mathrm{Br}\end{array} \\
\end{array}$} \\
\hline & I. & II. & \\
\hline C & $.37 \cdot 55$ & - & $37 \cdot 67$ \\
\hline H. & $5 \cdot 13$ & - & $4 \cdot 93$ \\
\hline $\mathrm{Br}$ & . - - & $36 \cdot 08$ & $35 \cdot 87$ \\
\hline
\end{tabular}

II. $0 \cdot 0989 \mathrm{~g}$ Substanz lieferte, mit Ätzkalk verbrannt, $0 \cdot 0834 \mathrm{~g}$ $\mathrm{AgBr}$.

In 100 Theilen:

Die durch Äther erschöpfte alkalische Lösung wurde eingeengt, wobei sich etwas Harz ausschied. Nach Abfiltriren von demselben wurde das Filtrat zur Trockene gebracht und nachdem darin Bromnatrium nachgewiesen war, mit Alkohol extrahirt. Die alkoholische Lösung wurde nach Zusatz von Wasser durch Abdampfen vom Alkohol befreit und mit Essigsäure neutralisirt. In der neutralen Flüssigkeit wurde Ameisensäure nachgewiesen, jedoch in relativ geringer Menge.

Die Einwirkung von Natriumcarbonat auf das Säuredibromid könnte demnach im Sinne der Gleichung 


$$
\begin{aligned}
2 \mathrm{C}_{7} \mathrm{H}_{12} \mathrm{O}_{3} \mathrm{Br}_{2}+2 \mathrm{Na}_{2} \mathrm{CO}_{3}=\mathrm{C}_{2} \mathrm{H}_{11} \mathrm{O}_{3} \mathrm{Br}+\mathrm{C}_{6} \mathrm{H}_{10} \mathrm{O}+3 \mathrm{NaBr}+ \\
+\mathrm{HCOONa}+2 \mathrm{CO}_{2}+\mathrm{H}_{2} \mathrm{O}+\mathrm{O}
\end{aligned}
$$

erfolgt sein, wobei möglicherweise der frei gewordene Sauerstoff noch oxydirend eingewirkt hätte theils auf $\mathrm{C}_{6} \mathrm{H}_{10} \mathrm{O}$, theils auf das ameisensaure Natrium. Ob sich aus der Säure Kohlensäure abgespalten hat, muss vorläufig dahingestellt bleiben. ${ }^{1}$

\section{Reduction der Säure.}

Die Versuche mit Natriumamalgam in alkalischer Lösung, mit Zink in salzsaurer und in essigsaurer Lösung die gesättigte Säure zu erhalten, ergaben wiederholt ein negatives Resultat.

Auch ein Versuch, mit Zink und Ammoniak zu reduciren, ergab als Endproduct nur die ungesättigte Säure, wie die in den einzelnen Fällen ausgeführte quantitative Addition von Brom bewies.

Dagegen führte die Reduction mit Jodwasserstoffsäure zu weit. Die gesättigte Säure entstand nicht, wohl aber unter Abspaltung von Ameisensäure gleichzeitig der gesättigte Kohlenwasserstoff $\mathrm{C}_{6} \mathrm{H}_{14}$ und das diesem entsprechende Jodid in je nach den Versuchsbedingungen vorherrschender Menge.

$4.10 \mathrm{~g}$ Säure wurden mit $0.3 g$ rothem Phosphor und $25 \mathrm{~cm}^{3}$ Jodwasserstoffsäure vom specifischen Gewichte 1.96 durch 16 Stunden hindurch im Rohr auf $150-160^{\circ}$ erhitzt. Nach dem Erkalten war obenauf eine ölige Schichte, die etwas heller als der übrige Rohrinhalt gefärbt war. Geringer Druck war beim Öffnen des Rohres wahrzunehmen und sehr deutlich ein petroleumartiger Geruch. Das obenauf schwimmende ölige Product wurde abgehoben und mit schwefeliger Säure und Wasser gewaschen. Es bildete eine schwach gelblich gefärbte Flüssigkeit, welche in Ätzkali unlöslich, schwerer als Wasser war und viel Jod enthielt. Die mit Chlorcalcium getrocknete Substanz wurde destillirt. Schon aus dem kochenden Wasserbad ging eine geringe Spur nach Petroleum riechend über. Die

1 Da ich weiterhin beschäftigt bin mit dem Studium der Einwirkung von Blausäure auf Tiglinaldehyd, hoffe ich seinerzeit die hier statthabende Reaction genauer untersuchen zu können. 
Hauptmenge destillirte im Vacuum bei $80-83^{\circ}$ und $32 \mathrm{~mm}$ Druck und ergab bei der Elementaranalyse und Jodbestimmung auf das Jodid $\mathrm{C}_{6} \mathrm{H}_{13} \mathrm{~J}$ gut stimmende Werthe:

I. $0 \cdot 1948 g$ Substanz ergaben, mit Bleichromat und vorgelegter Silberspirale verbrannt, $0.2417 \mathrm{~g} \mathrm{CO}_{2}$ und $0.1098 \mathrm{~g}$ $\mathrm{H}_{2} \mathrm{O}$.

II. $0 \cdot 1167 \mathrm{~g}$ Substanz lieferten nach Carius $0 \cdot 1288 \mathrm{~g} \mathrm{AgJ}$.

In 100 Theilen:

\begin{tabular}{|c|c|c|}
\hline \multicolumn{2}{|c|}{ Gefunden } & Berechnet \\
\hline I. & TI. & für $\mathrm{C}_{6} \mathrm{H}_{1: 3}{ }^{\mathrm{J}}$ \\
\hline$\ldots .33 \cdot 83$ & - & $33 \cdot 96$ \\
\hline $6 \cdot 22$ & - & $6 \cdot 13$ \\
\hline$\ldots \ldots-$ & $59 \cdot 62$ & $59 \cdot 90$ \\
\hline
\end{tabular}

Die nach Abheben der öligen Schichte im Rohr verbliebene Jodwasserstofflösung wurde mit gelbem Phosphor geschüttelt und destillirt. Die ersten Tropfen des Destillates enthielten eine ganz geringe Menge einer in Wasser unlöslichen, öligen Flüssigkeit, welche leichter als Wasser, einen sehr stark an Petroleum erinnernden Geruch zeigte und gewiss wohl der Kohlenwasserstoff $\mathrm{C}_{6} \mathrm{H}_{14}$ war, wie aus dem Folgenden geschlossen werden kann. Dieser Körper wurde abgehoben genügte jedoch zu keiner weiteren Untersuchung.

Das noch durch Jod gefärbte Destillat wurde wiederholt mit kleinen Stückchen gelben Phosphors geschüttelt und destillirt, bis schliesslich ein schwach saures, jedoch keine Jodwasserstoffsäure mehr enthaltendes Destillat resultirte, aus welchem das Baryumsalz dargestellt wurde. Es wurde qualitativ als ameisensaures Salz erkannt.

Bei einem anderen Reductionsversuch genügte es, $2 g$ Säure mit $12 \mathrm{~cm}^{3}$ Jodwasserstoffsäure vom specifischen Gewicht $1.67 \mathrm{im} \mathrm{Rohr} 10$ Stunden lang auf $150^{\circ} \mathrm{zu}$ erhitzen, um dasselbe Jodid zu erhalten.

Die Reaction lässt sich durch folgende Gleichung ausdrücken:

$$
\mathrm{C}_{7} \mathrm{H}_{12} \mathrm{O}_{3}+5 \mathrm{HJ}=\mathrm{C}_{6} \mathrm{H}_{13} \mathrm{~J}+\mathrm{HCOOH}+4 \mathrm{~J}+\mathrm{H}_{2} \mathrm{O} .
$$


Das Jodatom dürfte wohl an das in der ungesättigten Säure wasserstoffreie Kohlenstoffatom getreten sein, so dass für das analysirte Jodid die Formel

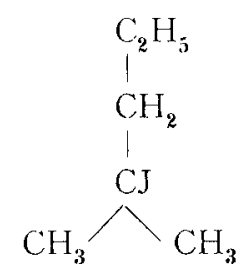

anzusprechen wäre.

Schon der Umstand, dass neben dem Jodid in geringer Menge ein Körper entstanden war, welcher leichter war als Wasser und ausgesprochen nach Petroleum roch, liess vermuthen, dass die Reduction theilweise bis zur Bildung von Kohlenwasserstoff gegangen war. Demnach war zu erwarten, dass beim Erhitzen auf noch höhere Temperatur der Kohlenwasserstoff selbst entstehen wird.

$3.0 \mathrm{~g}$ Säure wurden mit $0.3 \mathrm{~g}$ rothem Phosphor und $25 \mathrm{~cm}^{3}$ Jodwasserstoffsäure vom specifischen Gewichte 1.96 im Rohr eingeschlossen 16 Stunden lang auf $180-190^{\circ}$ erhitzt. Nach dem Erkalten war deutlich eine obere, heller gefärbte Schichte sichtbar. Beim Öffnen des erkalteten Rohres war der Druck ziemlich bedeutend und der petroleumartige Geruch auffallend stark. Kohlensäure war in dem entweichenden Gase nicht nachzuweisen. Die obere scharf von der Jodwasserstofflösung abgegrenzte Schichte wurde abgehoben, wie vorher mit schwefeliger Säure und Wasser gewaschen und mit Chlorcalcium getrocknet. Bei der Destillation aus dem zum Kochen erhitzten Wasserbad ging der weitaus grösste Antheil unter $67^{\circ}$ über, eine geringe Menge blieb zurück. Diese letztere enthielt viel Jod, dürfte also das früher beschriebene Jodid sein.

Nach wiederholter Destillation der Hauptmenge wurde der Siedepunkt bei $62-63^{\circ}$ gefunden. Das Product war farblos, leichter als Wasser, unlöslich in Ätzkali, zeigte Fluorescenz und roch ganz wie Petroleum. Brom wurde nicht aufgenommen. $0 \cdot 1060 \mathrm{~g}$ Substanz ergaben bei der Verbrennung $0 \cdot 3257 \mathrm{~g} \mathrm{CO}_{2}$ und $0 \cdot 1556 g \mathrm{H}_{z} \mathrm{O}$. 
In 100 Theilen:

$$
\begin{array}{ll}
\text { C..... } \ldots 83 \cdot 80 & \frac{\begin{array}{l}
\text { Berechnet } \\
\text { für } \mathrm{C}_{6} \mathrm{H}_{1-1}
\end{array}}{83 \cdot 72} \\
\mathrm{H} \ldots \ldots .16 \cdot 31 & 16 \cdot 28
\end{array}
$$

Die nach Meyer's Luftverdrängungsmethode bestimmte Dampfdichte ergab die Zahl 3.02, mithin ist das Moleculargewicht mit $87 \cdot 15$ gefunden, anstatt 86 für $\mathrm{C}_{6} \mathrm{H}_{\mathbf{1 4}}$.

Der nach Abheben der oberen Schichte verbliebene Rohrinhalt wurde in derselben Weise behandelt, wie früher bei der Darstellung des Jodid angegeben wurde. Eine ganz geringe Menge Kohlenwasserstoff wurde auch noch darin nachgewiesen und Ameisensäure.

Die Reactionsgleichung wäre:

$$
\mathrm{C}_{7} \mathrm{H}_{12} \mathrm{O}_{3}+6 \mathrm{HJ}=\mathrm{C}_{6} \mathrm{H}_{14}+\mathrm{HCOOH}+\mathrm{H}_{2} \mathrm{O}+6 \mathrm{~J} .
$$

Analyse, Dampfdichtebestimmung und die Unfähigkeit Brom zu addiren sprechen für den gesättigten Kohlenwasserstoff $\mathrm{C}_{6} \mathrm{H}_{14}$. Die Constitution ergibt sich aus der Entstehung desselben unter Abspaltung von Ameisensäure als ein in der x-Stellung methylirtes Pentan (4-Methyl-pentan).<smiles>CCCC(C)C</smiles>

Ein ebenso constituirtes Hexan wurde von $\mathrm{Wurtz}^{\mathbf{1}}$ aus Athyljodid und Isobutyljodid mit Natrium dargestellt mit dem Siedepunkte von $62^{\circ}$.

1 Jahresber., $1855,574$. 